\title{
ON THE LOWER ORDER OF AN ENTIRE DIRICHLET SERIES
}

\author{
J. P. SINGH
}

ABSTRACT. The lower order $\bar{\lambda}_{s}$ of $f(s)$ in each horizontal strip $S(\pi a)$, with $a>\Delta^{*}$, is equal to the lower order $\lambda$ of $f(s)$. The purpose of this note is to offer a proof of this result.

1. Let

$$
f(s)=\sum_{n=1}^{\infty} a_{n} e^{-\lambda_{n} s}, \quad s=\sigma+i t,
$$

be a function represented by a Dirichlet series convergent in the whole plane where $\left\{\lambda_{n}\right\}_{1}^{\infty} \uparrow_{\infty}$ is a sequence of positive, nondecreasing numbers with $\lim \inf _{n \rightarrow \infty}\left\{\lambda_{n+1}-\lambda_{n}\right\}>0$.

We shall use the following notations:

For a fixed $t_{0}$, let $S(R)$ denote the horizontal strip $\left|\bar{t}-t_{0}\right|<R$. Put

$$
\begin{gathered}
M(\sigma)=\operatorname{Max}_{-\infty<t<\infty}|f(\sigma+i t)|, \quad M_{s}(\sigma)=\underset{\left|t-t_{0}\right| \leq R}{\operatorname{Max}}|f(\sigma+i t)|, \\
\bar{M}_{s}\left(\sigma_{0}\right)=\operatorname{Max}_{\left|t-t_{0}\right| \leq R ; \sigma \geq \sigma_{0}}|f(\sigma+i t)|
\end{gathered}
$$

and let

$$
\begin{gathered}
\lim _{\sigma \rightarrow-\infty} \sup \frac{\log \log M(\sigma)}{-\sigma}=\frac{\rho}{\lambda} ; \quad \lim _{\sigma \rightarrow-\infty} \sup \frac{\log \log M_{s}(\sigma)}{-\sigma}={ }_{\lambda_{s}}^{\rho_{s}} ; \\
\lim _{\sigma_{0} \rightarrow-\infty} \sup \inf \frac{\log \log \bar{M}_{s}\left(\sigma_{0}\right)}{-\sigma_{0}}=\frac{\bar{\rho}_{s}}{\bar{\lambda}_{s} .}
\end{gathered}
$$

Further, following Malliavin [2], we shall denote the maximum, upper and lower logarithmic densities of $\left\{\lambda_{n}\right\}$ by $\Delta^{*}, \Delta^{0}$ and $\Delta_{0}$ respectively.

2. Mandelbrojt and Gergen [3, pp. 219-220] have proven that the order $\rho_{s}$ of $f(s)$ in each horizontal strip $S(\pi a)$, with $a>D$, is equal to the order $\rho$ of $f(s)$. This result has been extended to the lower order $\lambda_{s}$ by Rahman [4]. But the proof of his theorem is not complete. Further, Rahman [5] improved the proof of his theorem under the additional hypothesis (satisfied

Presented to the Society, May 10,1973; received by the editors November 27, 1972 and, in revised form, July 1, 1974.

AMS (MOS) subject classifications (1970). Primary 30A16, 30 A64.

Key words and phrases. Entire Dirichlet series, lower order, arithmetic density, logarithmic density. 
if the coefficients are positive) that enables the original proof to work, but the additional hypothesis is unnatural.

In this note, our object is to prove a theorem which is better and more precise than the theorems of Rahman [4], [5], Roux [6] and Srivastava [7]. In the proof, we use Malliavin's version [2, p. 232] of Mandelbrojt's fundamental inequality. This gives a sharper result, since Malliavin's inequality involves a logarithmic density that is finer than the arithmetic density of Mandelbrojt's inequality. We prove the following .

Theorem. The lower order $\bar{\lambda}_{s}$ of $f(s)$ in each horizontal strip $S(\pi a)$, with $a>\Delta^{*}$, is equal to the lower order $\lambda$ of $f(s)$.

3. Proof. It is known that there exists an increasing subsequence $\left\{n_{j}\right\}$ of $n$ for which

$$
\limsup _{j \rightarrow \infty} \frac{\log \left|1 / a_{n_{j}}\right|}{\lambda_{n_{j}} \log \lambda_{n_{j-1}}}=\frac{1}{\lambda}<\infty \quad[6] .
$$

Now, by Malliavin's version [2, p. 232] of Mandelbrojt's fundamental inequality, we get ${ }^{1}$

$$
\log \bar{M}_{s}\left(\sigma_{0}\right)>-(1 / \lambda+2 \epsilon) \lambda_{n_{j}} \log \lambda_{n_{j}-1}-\sigma_{0} \lambda_{n_{j}}-\lambda_{n_{j}}\left[k\left(\lambda_{n_{j}}\right)-k \cdot\left(\lambda_{n_{j}}\right)\right] .
$$

Since for sufficiently large $x$,

$$
2\left(\Delta_{0}-\epsilon\right) \log x<\lambda(x)<2\left(\Delta^{0}+\epsilon\right) \log x
$$

and $k(x)=2 a \log x-\lambda(x)$, hence

$$
\begin{gathered}
2\left(a-\Delta^{0}-\epsilon\right) \log x<k(x)<2\left(a-\Delta_{0}+\epsilon\right) \log x, \\
k \cdot(x)>2\left(a-\Delta^{0}-\epsilon\right) \log x .
\end{gathered}
$$

We have

$$
A=\limsup _{x \rightarrow \infty} \frac{k(x)-k \cdot(x)}{\log x} \leq 2\left(\Delta^{0}-\Delta_{0}\right) .
$$

Under the hypothesis of the Theorem $\Delta^{0}=\Delta_{0}$, therefore, we get from (3.1)

$$
\log \bar{M}_{s}\left(\sigma_{0}\right)>-\left[(1 / \lambda+3 \epsilon) \log \lambda_{n_{j-1}}+\sigma_{0}\right] \lambda_{n_{j}}
$$

Choose $\sigma_{j+1}=-(1 / \lambda+4 \epsilon) \log \lambda_{n_{j}}$. For any $\sigma_{0}$ satisfying the inequalities $\sigma_{j+1}<\sigma_{0} \leq \sigma_{j}, \bar{M}_{s}\left(\sigma_{0}\right)$ is decreasing for increasing $\sigma_{0}$. Hence, we have

$$
\bar{\lambda}_{s}=\liminf _{\sigma_{0} \rightarrow-\infty} \frac{\log \log \bar{M}_{s}\left(\sigma_{0}\right)}{-\sigma_{0}} \geq \liminf _{j \rightarrow \infty} \frac{(1+o(1)) \log \lambda_{n_{j}}}{(1 / \lambda+4 \epsilon) \log \lambda_{n_{j}}}=\frac{1}{(1 / \lambda+4 \epsilon)} .
$$

\footnotetext{
${ }^{1}$ Notations, used here, are same as Malliavin's [2].
} 
Since $\epsilon$ is arbitrary, $\bar{\lambda}_{s} \geq \lambda$. But $\bar{\lambda}_{s} \leq \lambda$ always. The case $\lambda=0$ is obvious. This leads to the desired conclusion.

4. Remarks。1. The errors and omissions in the proof of Rahman's theorem were pointed out by Sungar $i$ Balaguer [8], but he did not provide the proof, of Theorem 1 of [4].

2. Our definition of $\bar{\lambda}_{s}$ is slightly different from those used in [4], [5] and [7].

3. Roux [6] has used a different definition of lower order in the strip (see Blambert [1]).

The author thanks Dr. Shankar Hari Dwivedi and Dr. V. B. Goyal for their continuous encouragement during the preparation of this paper and also wishes to express his thanks to the referee for his help in its preparation.

\section{REFERENCES}

1. M. Blambert, Sur la notion de type de l'ordre d'une fonction entière, Ann. Sci. Ecole Norm. Sup。 (3) 79 (1962), 353-375. MR 31 \#3606.

2. P. Malliavin, Sur quelques procédés d'extrapolation, Acta Math. 93 (1955), 179-255. MR 17, 724.

3. S. Mandelbrojt, Dirichlet series, Rice Inst. Pamphlet 31 (1944), 157-272. MR 6, 267.

4. Q. I. Rahman, $O n$ entire functions defined by a Dirichlet series, $\dot{P}_{\text {roc. Amer. }}$ Math. Soc. 10 (1959), 213-215. MR 23 \#A299.

5. $\longrightarrow$ On entire functions defined by a Dirichlet series: Correction, Proc. Amer. Math. Soc. 11 (1960), 624-625. MR 23 \#A300.

6. D. Roux, Sull'ordine inferiore delle funzioni intere, Boll. Un. Mat. Ital. (3) 20 (1965), 379-388. MR 33 \#2815.

7. K. N. Srivastava, On the lower order of an entire Dirichlet series, Math. Japon. 5 (1958/59), 109-112. MR 23 \#A1784.

8. F. Sunyer i Balaguer, $O_{n}$ entire functions defined by a Dirichlet series, Proc. Amer. Math. Soc. 11 (1960), 621-623. MR 23 \#A301.

DEP ARTMENT OF MATHEMATICS, KURUKSHETRA UNIVERSITY, KURUKSHETRA132119 INDI A 